\title{
$H$. pylori infection and gastric cancer: State of the art (Review)
}

\author{
VINCENZA CONTEDUCA ${ }^{1}$, DOMENICO SANSONNO ${ }^{1}$, GIANFRANCO LAULETTA ${ }^{1}$, \\ SABINO RUSSI $^{1}$, GIUSEPPE INGRAVALLO ${ }^{2}$ and FRANCO DAMMACCO ${ }^{1}$ \\ Departments of ${ }^{1}$ Internal Medicine and Clinical Oncology and ${ }^{2}$ Pathology, \\ University of Bari ‘Aldo Moro’ Medical School, Bari, Italy
}

Received September 18, 2012; Accepted October 29, 2012

DOI: $10.3892 /$ ijo.2012.1701

\begin{abstract}
Gastric cancer (GC) is one of the leading types of cancer worldwide, particularly in East Asian populations. Helicobacter pylori (HP) infection has been established as a major risk factor for GC. Although more than $50 \%$ of the world population is infected with this bacterium, less than $2 \%$ develop GC. Therefore, further risk factors (such as host genetic polymorphisms and lifestyle, as well as environmental and epigenetic factors) may also play a role in its occurrence. The correlation between HP infection and GC represents a typical model of a multi-step process, characterized by some pre-neoplastic lesions with a high risk of progression (atrophic gastritis, intestinal metaplasia and dysplasia). In addition, HP also plays an oncogenic role in the development of mucosa-associated lymphoid tissue (MALT) lymphoma, that accounts for approximately 3\% of all gastric tumors. Hyperplastic polyps often arise in patients with atrophic gastric mucosa and HP-associated gastritis $(25 \%$ of cases); however, their malignant trasformation is rare $(<3 \%$ of cases). A number of trials have demonstrated the possibility of cancer prevention through HP screening and eradication, particularly in high-risk populations, whereas it may not be cost-effective in areas of low risk. In this review, we discuss i) the complex pathogenetic mechanisms of gastric carcinogenesis in which HP is involved; ii) the main approaches to the diagnosis, prevention, surveillance and treatment of pre-malignant lesions associated with HP infection; iii) the most effective way to detect
\end{abstract}

Correspondence to: Dr Vincenza Conteduca, Liver Unit, Department of Internal Medicine and Clinical Oncology, University of Bari 'Aldo Moro' Medical School, Piazza G. Cesare 11, I-70124 Bari, Italy

E-mail: cinzia.conteduca@libero.it

Abbreviations: BabA, blood group antigen-binding adhesion; cag, cytotoxin-associated gene; COX-2, cyclo-oxygenase-2 (COX-2); GC, gastric cancer; HP, Helicobacter pylori; IL, interleukin; MALT, mucosa-associated lymphoid tissue; NHL, non-Hodgkin's lymphoma; OipA, outer inflammatory protein A; cag PAI, cag pathogenicity island; PGE2, prostaglandin E2; RUNX3, Runt-related transcription factor 3; SCK, Src tyrosine kinase; VacA, vacuolating toxin-A; VEGF, vascular endothelial growth factor

Key words: endoscopic surveillance, gastric cancer, gastric polyps, Helicobacter pylori, Helicobacter pylori eradication, mucosaassociated lymphoid tissue lymphoma
GC in its earlier stages; and iv) the most important contribution to reducing the burden of GC.

\section{Contents}

1. Epidemiology

2. Etiology

3. Gastric polyps

4. Diagnosis

5. Screening and surveillance

6. Prevention and treatment

7. Conclusions

\section{Epidemiology}

Gastric cancer (GC) is the second leading cause of cancerrelated mortality worldwide, with an estimated 989,600 new cases (male-to-female ratio 2:1) and 738,000 GC-related deaths in 2010, accounting for $8 \%$ of the total cancer cases and $10 \%$ of total deaths from cancer. Moreover, $>70 \%$ of these new cases and the GC-related deaths occur in developing countries, particularly in Eastern Asia (1).

The most common histopathological features of gastric malignancies are adenocarcinoma and lymphoma of mucosaassociated lymphoid tissue (MALT). Approximately $90 \%$ of gastric tumors are adenocarcinomas, whereas gastric MALT lymphomas are considerably less common (approximately 3\% of all gastric tumors). MALT lymphoma is a distinct subtype of marginal zone B-cell non-Hodgkin's lymphoma (NHL), accounting for approximately $7-8 \%$ of all NHLs, with the gastrointestinal tract being the most common site of the disease (2).

Helicobacter pylori (HP) infection plays an important cancerogenic role in both gastric carcinoma and MALT lymphoma. Indeed, HP is estimated to colonize the stomach in approximately one-half of the world population, with a variable prevalence in different countries (3). For example, in Eastern regions of Asia and in some parts of Latin America, HP infection occurs at an early age, usually in childhood, resulting in approximately $80 \%$ of the population being infected by the age of 20. By contrast, in developed countries such as France, the USA, the UK, or Australia, the prevalence of HP infection is low in children under the age of 10 years but increases to approximately $40 \%$ in adults $30-40$ years of age. In the USA, 
the prevalence of HP infection varies among the different subpopulations and is several-fold higher in the children of ethnic groups, such as Afro-Americans and Asians, with lower socioeconomic status. Moreover, migrant populations from high-risk areas, including Japan, show a marked risk reduction when they move to low-incidence regions, such as the USA, and subsequent generations acquire risk levels comparable to those of the host country. Thus, the difference in prevalence among ethnic groups of similar socioeconomic status possibly reflects environmental causes and host genetic alterations.

It has been calculated that the risk of gastric adenocarcinoma and MALT lymphoma in HP-infected individuals is 3- to 6 -fold higher than in those who are uninfected (2). The association of HP infection with gastric carcinoma mainly involves intestinal-type and distal forms of the malignancy. There are 2 main sites of gastric adenocarcinoma: proximal (cardia) and distal (non-cardia), with different epidemiological and clinical features. In non-cardia GC, other risk factors have been implicated, including low socioeconomic status, smoking, salty and smoked food intake, low consumption of fruits and vegetables and a family history of GC (4). In contrast to the decline in distal GCs, there has been a progressive increase in proximal tumors since the 1970s, particularly among males in Western countries. Gastric cardia cancers share certain molecular profiles with adenocarcinomas of the distal esophagus and gastro-esophageal junction, suggesting that they represent a similar disease entity. In fact, the main risk factors for all of these tumors are obesity, gastro-esophageal reflux disease and Barrett's esophagus (5).

Histologically, GCs are subdivided into 2 main types (Lauren classification): i) intestinal type; and ii) undifferentiated or diffuse type. GC does not arise from a normal mucosa. The intestinal type is related to corpus-dominant gastritis with intestinal metaplasia, whereas the diffuse type usually originates from superficial pangastritis without atrophy. In addition, while the former is more common in males, blacks and older age groups, the latter has a comparable male-to-female ratio and is more frequent in younger individuals. Intestinal-type tumors predominate in high-risk geographical areas ('epidemic type'), such as Eastern Asia, Eastern Europe and Central and South America. Diffuse-type carcinomas of the stomach have a more uniform geographical distribution ('endemic type'). However, the incidence of diffuse-type gastric carcinoma, in particular signet ring-type, has been increasing (6).

Thus, GC incidence rates vary by up to 10 -fold worldwide. Regional variations in part reflect differences in dietary patterns, particularly in European countries and in the prevalence of HP infection. Gastric colonization by HP is usually asymptomatic and, although in approximately $20 \%$ of infected populations the bacterium is responsible for pre-neoplastic changes, gastric neoplasms develop in $<2 \%$. Gastric lymphoma is an even rarer consequence of HP infection, occurring in $<1 \%$ of infected individuals (7) (Fig. 1).

GC rates have substantially decreased in most geographical areas, possibly due to the increased use of refrigeration, the availability of fresh fruits and vegetables and the decreased consumption of salted and preserved foods. Despite the potentially low malignant transformation of gastric pre-cancerous lesions induced by HP, the eradication of this bacterial infection may reduce the risk of gastric adenocarcinoma and gastric MALT lymphoma.
In fact, the regression of gastric MALT lymphoma occurs in $60-80 \%$ of HP-positive patients, suggesting HP eradication as the first-choice treatment (8). In a meta-analysis, the relative risk of GC following HP eradication was calculated to be 0.65 overall (9). Nevertheless, GC still implies a poor prognosis and high mortality. In general, patients living in countries with a higher incidence of GC have better survival rates than patients from countries with a lower incidence. This association is mainly due to the location of the tumor within the stomach. Patients with tumors located in the gastric cardia (which are more frequent in areas with a lower incidence) have a much poorer prognosis than those with tumors arising in the pyloric antrum (more frequent in areas with a higher incidence), with lower 5-year survival rates and higher operative mortality. In addition, accurate screening for the early detection in high-risk areas has led to a reduction in mortality. In Japan, mortality rates for males with GC have markedly decreased over the past several decades, due to mass screening programs.

When the disease is confined to the inner lining of the gastric wall, the 5-year survival is approximately $95 \%$. Unfortunately, the majority of GCs are not discovered at an early stage, thus leading to 5-year relative survival rates of $<20 \%$. Therefore, early detection is a crucial prognostic factor. Patients with a family history of non-hereditary GC have a higher risk of developing these tumors. Familial clusters of stomach cancer reflect a shared exposure to environmental hazards and to inherited factors. GC is associated with inherited syndromes in 1-3\% of cases. E-cadherin mutations are more frequent in hereditary syndromes, occurring in approximately $25 \%$ of families with autosomal dominant hereditary diffuse GC (HDGC), which arises as a result of germline mutations in the E-cadherin/DCH1 gene (10).

\section{Etiology}

Gastric carcinogenesis is a multifactorial process in which HP infection is the most important risk factor. Host genetic features, such as a pro-inflammatory cytokine profile and/or a positive family history, as well as bacterial virulence, further increase the risk of GC development. Other environmental issues, such as nutrition and socioeconomic conditions, are additional important influences.

The correlation between HP infection and the occurrence of GC represents a model of cancer development as a consequence of a microbial infection and chronic inflammation. The World Health Organization (WHO) has classified HP as a class I carcinogen since 1994 (11). The mechanisms whereby HP significantly increases the risk of gastric adenocarcinoma are clearer for intestinal-type GC, which progresses in a well-defined series of histological steps. The development of this type is marked by a slow progression, beginning with HP infection and subsequently progressing to chronic active gastritis, which occurs in all infected individuals. Under the influence of variable environmental and host factors, chronic active gastritis may in turn evolve into atrophic gastritis and intestinal metaplasia. In certain individuals, the metaplastic epithelium undergoes further genomic and phenotypic changes, resulting in gastric dysplasia and, finally, in adenocarcinoma. Variable progression rates to GC ranging from 0 to $2 \%$ per year have been reported in patients with atrophic gastritis. However, 


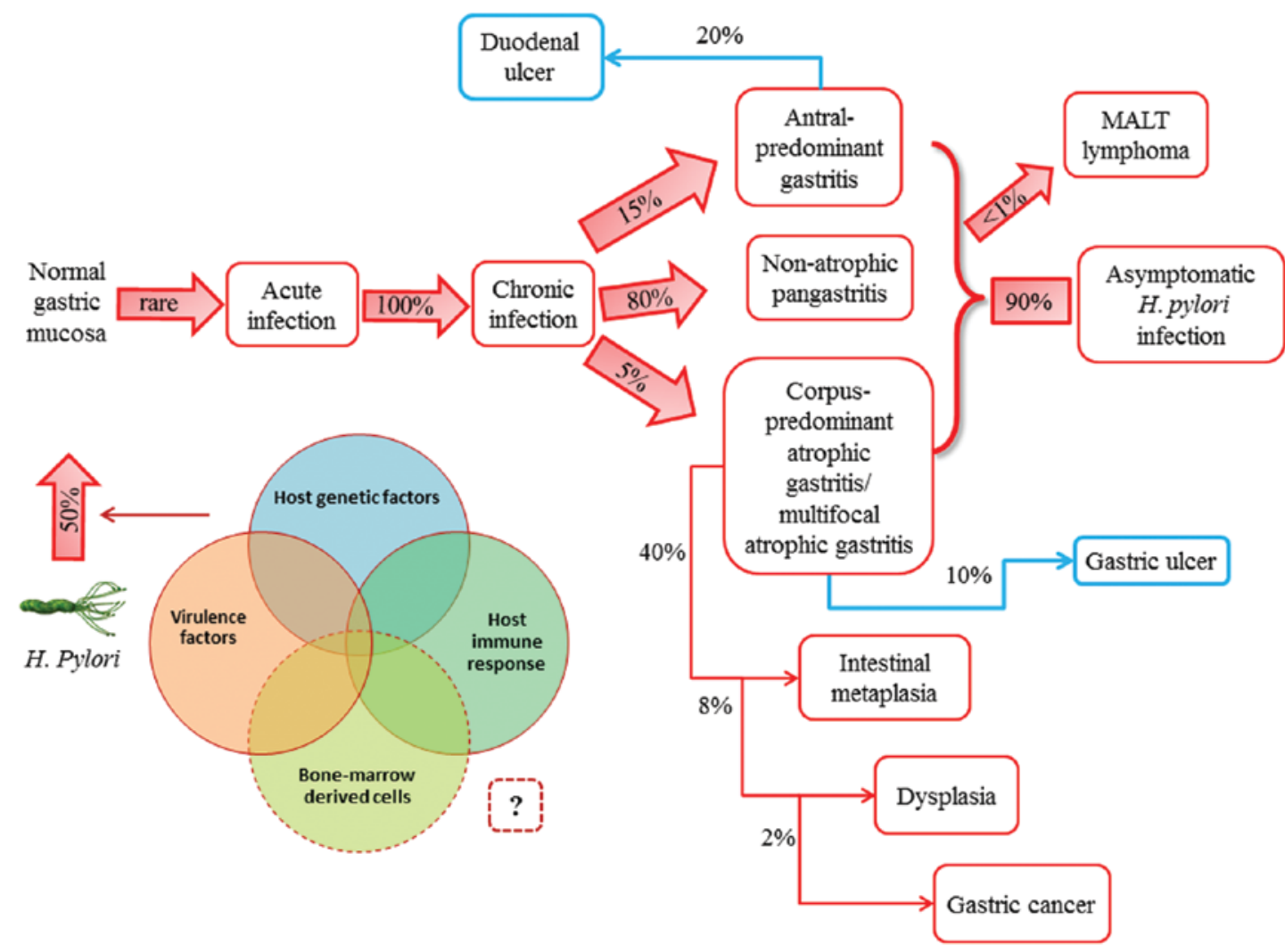

Figure 1. Natural history of Helicobacter pylori (H. pylori) infection. H. pylori is usually acquired in childhood, whereas acute infection with the bacterium is rarely diagnosed. Instead, chronic gastritis develops in almost all persistently colonized individuals, $90 \%$ of whom will remain asymptomatic. The clinical course of $H$. pylori infection is highly variable depending on bacterial and host (genetic and immune) factors. Recent studies have supported the possible role of bone marrow-derived cells (i.e., gastric stem cells) in tumor progression. Patients with increased acid secretion are more likely to have antral-predominant gastritis, which predisposes to duodenal ulcers. Patients with low acid secretion will more likely develop gastritis in the body of the stomach and are thus more likely to develop gastric ulcer, leading to gastric atrophy, intestinal metaplasia, dysplasia and, finally, in rare cases, gastric carcinoma. This sequence of events is more frequent in people of advanced age. H. pylori infection induces the formation of mucosa-associated lymphoid tissue (MALT) in the gastric mucosa and MALT lymphoma is another rare complication of $H$.pylori infection.

progression rates from intestinal metaplasia and dysplasia to GC are widely variable, ranging per year from 0 to $10 \%$ and from 0 to $73 \%$, respectively (12).

Prior to the discovery of HP, in 1988, Correa proposed a multistep cascade leading to GC (13). This model has been clearly reproduced in Mongolian gerbils infected with HP (14). This Gram-negative spiral-shaped bacterium has unipolar flagella that helps it to colonize the stomach. As noted above, infection is usually acquired during infancy and it typically induces a life-long chronic gastritis. HP is specifically adapted to survive in the hostile acidic gastric environment, with gastric colonization resulting in the development of gastritis in virtually all infected individuals. The adhesion of the bacteria to epithelial cells induces an inflammatory response, resulting in the recruitment of neutrophils, followed by B and T lymphocytes, macrophages and plasma cells. Consequently, large amounts of reactive oxygen or nitrogen species, involved in epithelial cell damage and carcinogenesis, are generated (15). The major virulence factors of HP with a well-established role in the induction of mucosal inflammation include the cytotoxin-associated gene (cag) pathogenicity island (PAI)-encoded virulence factors, such as the cytotoxin-associated antigen (CagA) protein, the vacuolating toxin-A (VacA), the blood group antigen-binding adhesin (BabA) and the outer inflammatory protein (OipA). These proteins are encoded in a 40-kilobase segment of DNA that includes a group of approximately 30 genes, including those for type IV secretion system components.

CagA, encoded by cag PAI, is translocated into the epithelial cytosol. This cytotoxin is a 121 - to $145-\mathrm{kDa}$ immunodominant protein that is commonly considered a putative bacterial oncoprotein. In fact, it has been used as a marker for epidemiological studies of GC. Within Western populations, CagA-positive strains are more commonly associated with peptic ulceration, atrophic gastritis and gastric adenocarcinoma than cag-negative strains. Conversely, in many populations with a high incidence of GC, such as the Eastern regions of Asia, almost all HP strains are cag-positive (16).

Epithelial cells recognize the translocated CagA as a signaling molecule that is activated following tyrosine phosphorylation by Src kinases. This form interacts with the tyrosine phosphatase SHP-2, the C-terminal Src tyrosine kinase (SCK) and the adaptor protein $\mathrm{Crk}$, together resulting in cytoskeletal reorganization and cell elongation. In turn, these changes lead to cell scattering and so-called 'hummingbird' morphological changes. They also induce MAP kinase signaling, resulting in abnormal cell proliferation by promoting cell cycle progression. The latter finding, together with the phenotypic changes, demonstrates that CagA-activated SHP-2 plays an important role in cell transformation and GC promotion. Phosphorylated CagA binds the adaptor protein Crk, leading to cytoskeletal 
reorganization, the disruption of epithelial cell tight junctions and tissue damage. Non-phosphorylated CagA also interacts with certain host cell proteins, such as epithelial tight junctions, the hepatocyte growth factor receptor C-Met, E-cadherin/ $\beta$-catenin, the adaptor protein GRB-5 and kinase PAR1. These CagA-host-protein interactions disrupt tight and adherent junctions, leading to a loss of cell polarity and inducing pro-inflammatory and mitogenic effects that may be important in gastric carcinogenesis.

As a consequence of direct interactions between CagA and E-cadherin, the formation of E-cadherin/ $\beta$-catenin complexes is impaired and cytoplasmic and nuclear accumulations of $\beta$-catenin ensue. Downstream events include the transcription of genes involved in intestinal differentiation, such as cdx1/cdx 2 and the muc2 mucin gene, causing trans-differentiation from gastric to intestinal-type epithelial cells (17). HP stimulates gastric epithelial cells to express and release excessive amounts of pro-inflammatory cytokines, including interleukin-8 (IL-8) and IL-1. Pro-inflammatory IL-1 gene cluster polymorphisms (IL-1B, encoding IL-1B and IL-IRN, encoding its naturally occurring receptor antagonist) increase the risk of both intestinal- and diffuse-types of non-cardia GC, while causing a reduction in gastric acid secretion, stimulating hypergastrinemia and promoting mucosal damage in atrophic gastritis. Thus, a high-risk IL-1 genotype increases the likelihood of non-cardia GC, a disease that is characterized by hypochlorhydria, while it has no effect on cancers associated with high-level acid exposure, such as esophageal adenocarcinoma and certain gastric cardia cancers (18).

Another major HP virulence factor is VacA. After entering the epithelial cell membrane, it induces vacuole formation and exerts mitochondrial effects, leading to apoptosis. The polymorphism is also found among the VacA alleles and results in different levels of cytotoxicity (7). The detection and characterization of HP CagA and VacA genotypes may be useful for the identification of patients with gastric pre-neoplastic lesions who are at a high risk of disease progression and therefore need more intensive surveillance (19).

The HP OipA, if expressed together with CagA, is associated with an enhanced inflammatory response in the gastric mucosa $(18,19)$. OipA is universally present in HP strains in Eastern Asian populations but in $<50 \%$ of all strains in Western countries. Functional receptors for HP adhesion to the gastric epithelial cell surface include fucosylated ABO blood group and Lewis $\mathrm{b}$ antigens. BabA is an outer membrane protein encoded by the BabA2 gene; it binds to the Lewis $b$ antigen, $\mathrm{ABO}$ antigens and the sialyl-Lewis $\mathrm{x} / \mathrm{a}$ antigens.

The BabA-mediated adhesion of HP to the gastric mucosal cells facilitates HP colonization, induces mucosal inflammation and promotes the expression of sialyl-Lewis x/a (17). Moreover, CagA-positive HP infection upregulates cyclo-oxygenase-2 (COX-2) expression in gastric mucosa and in cancer. COX-2 is usually undetectable in normal tissue but becomes abundant at sites of inflammation and may be overexpressed in gastric carcinomas. The overexpression of COX-2 leads to an increase in the synthesis and release of prostaglandins, such as PGE2. This COX-2-induced prostaglandin pathway promotes carcinogenesis by increasing cell proliferation, inhibiting apoptosis and enhancing the invasiveness of malignant cells. HP infection is thought to induce $\mathrm{COX}-2$ expression in pre-cancerous gastric lesions, in turn upregulating the expression of vascular endothelial growth factor (VEGF) and promoting angiogenesis. COX-2 expression significantly decreases following HP eradication in patients with atrophic gastritis $(15,20)$.

Functional polymorphisms of toll-like receptor 4 (TLR4), a cell-surface lipopolysaccharide (LPS) receptor involved in HP recognition and host response, have been associated with an increased grade of inflammation and severe tissue damage in HP-infected individuals. Specifically, carriers of the TLR4+896A > G polymorphism have more severe gastric atrophy and inflammation as well as an increased risk of non-cardia GC (21). Finally, polymorphisms and the genetic diversity of essential pathogenic elements, such as the CagA, TRL4 and SHP2 genes appear to influence the oncogenic potential of HP strains (15).

The ability to stimulate gastrin production is an important aspect of HP-associated cancerogenesis. In transgenic mice, hypergastrinemia is associated with the overexpression of certain growth factors, the COX-2-prostaglandin system and anti-apoptotic proteins, such as survivin and $\mathrm{Bcl}-2$, leading to the proliferation of mutated atrophic cells, enhanced angiogenesis and the development of gastric tumors (Fig. 2).

A recent study demonstrated the causal role of HP infection as well as specific alterations of DNA methylation patterns in the gastric mucosa of HP-infected patients and in GC cell lines in vitro (22). Genes specifically methylated during HP infection include E-cadherin (CDH1), a member of the transmembrane glycoprotein family expressed by epithelial tissues. The CDH1 protein not only acts as a cell adhesion molecule, but also plays an important role in cellular growth and carcinogenesis. The importance of the CDH1 gene in diffuse-type GC is suppported by the finding that germline mutations in $\mathrm{CDH} 1$ are responsible for the HDGC syndrome and are commonly acquired in sporadic GC associated with HP infection. Inactivation of E-cadherin correlates with the infiltrative and metastatic potential of GC. Patients with E-cadherin-positive GCs have significantly longer 3- and 5-year survival rates than patients with E-cadherin-negative tumors (4). The methylation of the E-cadherin promoter can be reversed by the eradication of HP (3).

The methylation of the tumor suppressor, Runt-related transcription factor 3 (RUNX3), by HP infection likewise contributes to GC progression. Circulating RUNX3 methylation may therefore be a valuable biomarker for the detection of early GC (23).

$\mathrm{HP}$ is considered the initiator of a chronic inflammatory response that recruits bone marrow-derived cells to the gastric mucosa, thereby contributing directly to GC (24). HP also plays an important role in gastric MALT lymphomagenesis, favoring infection-associated indirect lymphoid transformation. In addition to its chronic persistence in host tissues, HP can trigger a sustained lymphoid proliferation, which provides a selective advantage to lymphoid clones that still remain dependent upon antigen stimulation. The evolution into malignant NHL occurs in the presence of additional oncogenic events, such as the constitutive activation of signaling pathways following chromosomal translocations, including $\mathrm{t}(1 ; 14)$ and $\mathrm{t}(14 ; 18)$, or the inactivation of tumor-suppressor genes by hyper-methylation or mutations, causing the lymphoproliferation to become independent of antigenic stimulation. Usually, mutations in p53 are associated with the transformation of low-grade into high-grade NHL (25) (Fig. 3). 


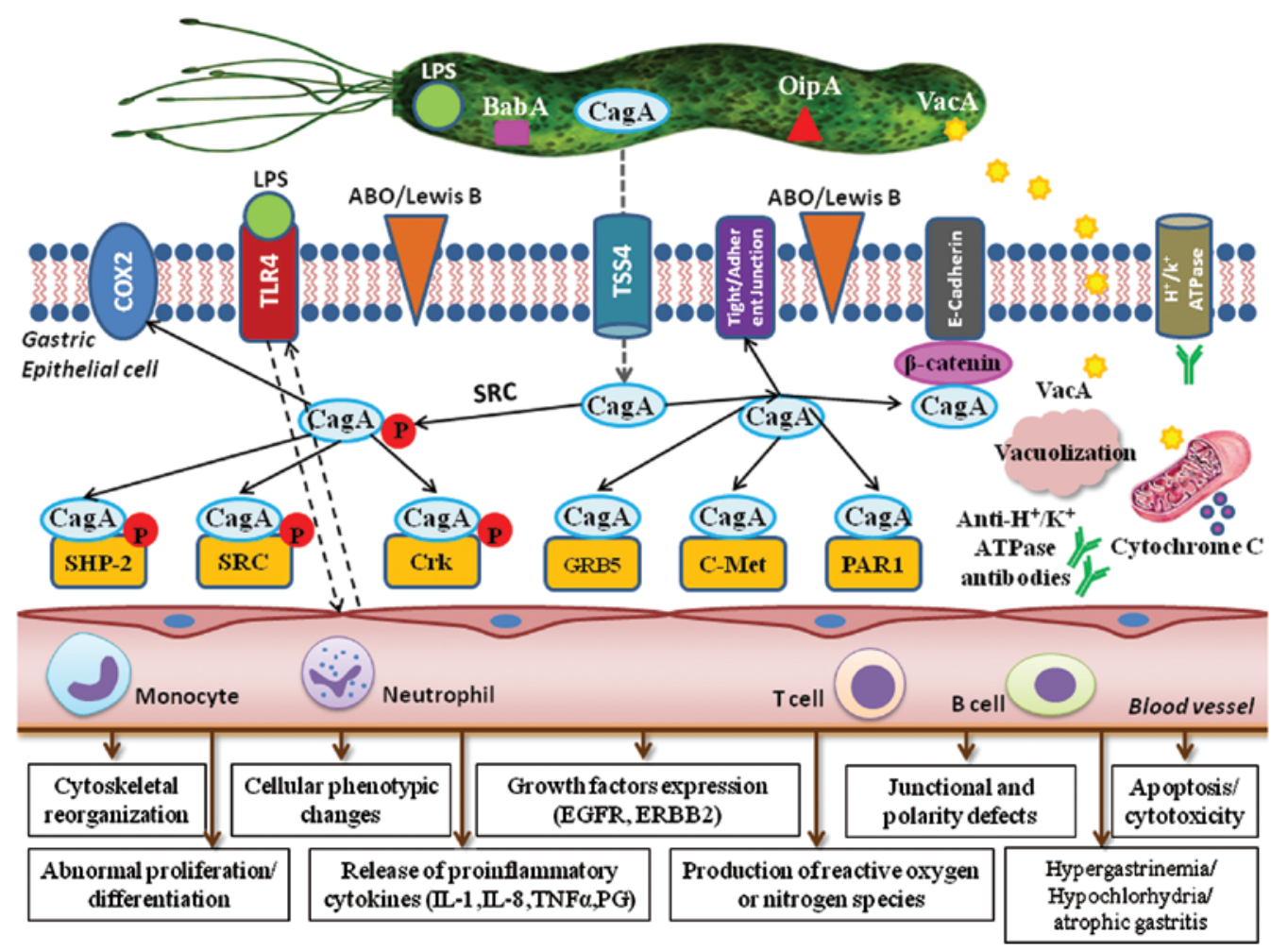

Figure 2. Pathogenesis of Helicobacter pylori (H.pylori) infection. Several virulence factors, such as CagA and VacA, encoded by $H$.pylori genes, interact with gastric epithelial cells and the immune system, resulting in an inflammatory response, mucosal damage and, eventually, gastric cancerogenesis (see description in the text).

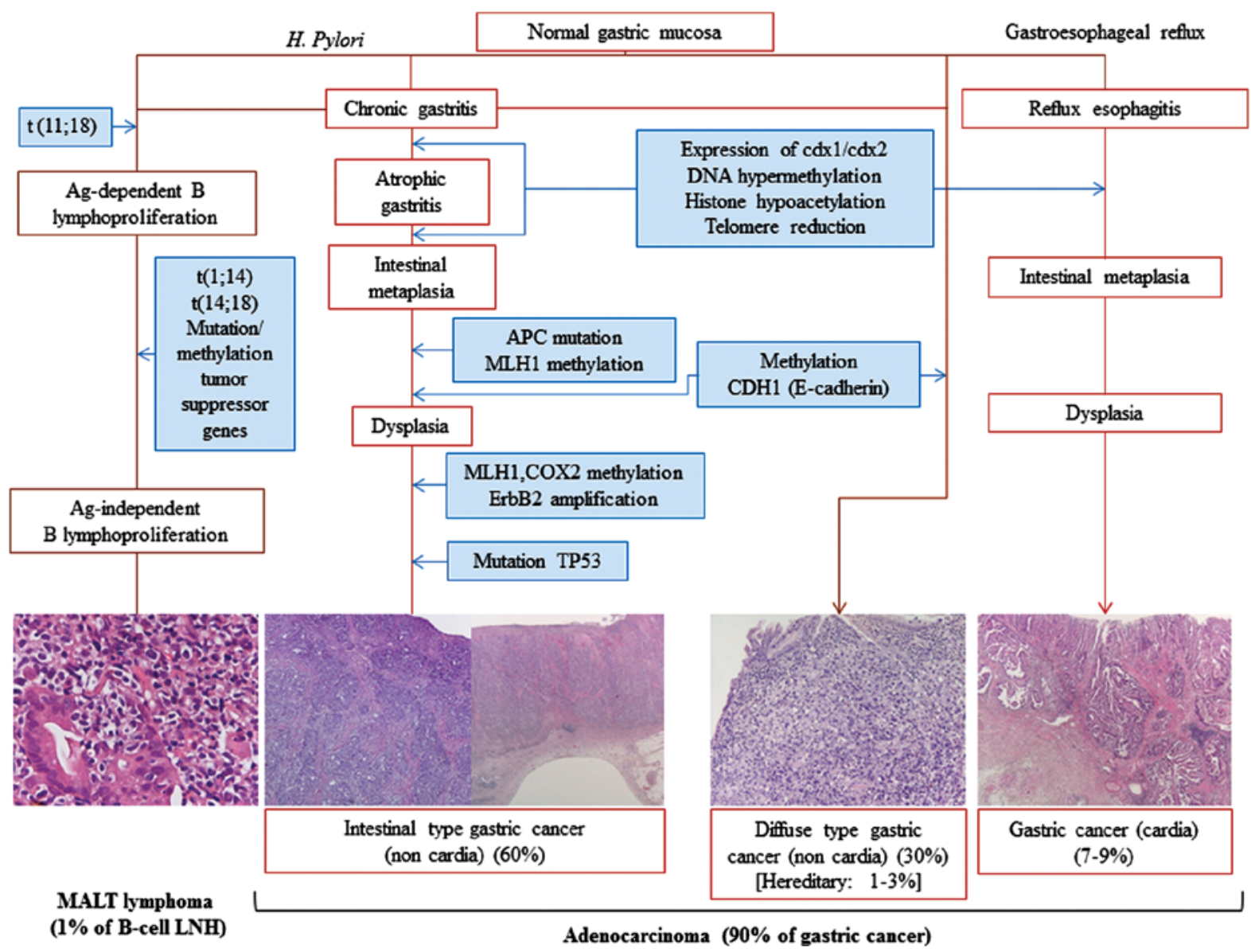

Figure 3. Multiple genetic and epigenetic alterations during gastric carcinogenesis and lymphomagenesis and the incidence rates in different types of gastric tumors. 


\section{Gastric polyps}

Some gastric polyps may have malignant potential and are associated with synchronous gastric adenocarcinomas. Approximately 1-4\% of patients who undergo gastric biopsy have gastric epithelial polyps. Adenomatous polyps have a higher rate of malignant transformation, whereas the risk of malignant transformation is very low for hyperplastic gastric polyps and virtually absent in sporadic fundic gland polyps. Therefore, the diagnosis of an adenomatous gastric polyp may support the inclusion of the patient in an endoscopic surveillance program, while only limited follow-up is generally proposed for patients with diagnoses of hyperplastic polyps, inflammatory polyps, or sporadic fundic gland polyps (26).

Adenomatous polyps may occur sporadically or in association with a familial adenomatous polyposis syndrome. Endoscopically, these polyps are lobulated in appearance, usually solitary (80\%), located in the antrum and $<2 \mathrm{~cm}$ in diameter. They may form circumscribed lesions, or they may be pedunculated or sessile. Histology will reveal a dysplastic epithelium without detectable invasion of the lamina propria. The prevalence of adenomatous polyps varies widely and is estimated to be $0.5-3.7 \%$ in Western countries and 9-27\% in areas with higher rates of GC, such as China and Japan. The larger the adenomatous polyp, the greater the probability of malignant evolution, particularly when foci of adenocarcinoma are present.

Hyperplastic polyps often arise in patients with an atrophic gastric mucosa and HP-associated gastritis (25\% of cases). They account for $18-70 \%$ of gastric polyps and are more frequently detected in the antrum than in other parts of the stomach. Usually, these polyps are multiple, smooth and small in diameter (measuring $0.5-1.5 \mathrm{~cm}$ ), while dysplasia is rare (<3\% of cases) (27).

Fundic gland polyps are the most common type of polyps detected in patients undergoing esophagogastroduodenoscopy in Western countries, with a prevalence of $13-77 \%$. Endoscopically, they appear as smooth, glassy, sessile, circumscribed elevations (usually measuring $<0.5 \mathrm{~cm}$ ) in the oxyntic mucosa. Fundic gland polyps may occur sporadically, in association with the use of proton-pump inhibitors, or in patients with familial adenomatous polyposis syndrome. They may or may not be associated with HP infection. In patients who use proton-pump inhibitors, fundic gland polyps may regress after the interruption of therapy.

\section{Diagnosis}

The symptoms of gastric pre-malignant and malignant diseases are usually non-specific and vague, such that early diagnosis is very difficult and GC is often diagnosed at an advanced stage. However, dyspeptic symptoms may occur in approximately $60-90 \%$ of patients with gastric pre-malignant lesions. Dyspepsia is defined by the presence of one or more symptoms of epigastric pain, burning, postprandial fullness, or early satiation. Bloating and nausea often coexist with dyspepsia but are non-specific and are thus not included in its definition. Heartburn is also excluded from the diagnostic criteria of dyspepsia since it is thought to primarily arise from the esophagus and is suggestive of gastro-esophageal reflux disease, although it too may occur concomitantly. These symptoms are generally indistinguishable from gastric malignant disease. It is therefore crucial to consider the diagnosis of gastric pre-malignant or malignant cancer in symptomatic patients.

Upper endoscopy is performed as the initial diagnostic test in patients with unexplained and persistent dyspepsia who are considered to be at higher risk. They are over 45 years of age (this cut-off was set since GC below the age of 45 is rare and its occurrence may vary between countries, depending on the prevalence of GC) and/or complain of other alarming features (anemia or evidence of acute/chronic bleeding, odynophagia, dysphagia, recurrent or persistent vomiting, unintentional weight loss, previous history of peptic ulcers).

Once the cause of any alarming features, typical gastroesophageal reflux disease symptoms and possible offending medications [such as non-steroidal anti-inflammatory drugs (NSAIDs), COX-2 inhibitors, iron, bisphosphonates, erythromycin, tetracycline, potassium supplements, acarbose, digitalis, theophylline] has been excluded in dyspeptic patients under the age of 45, evaluation for HP infection is warranted. Currently, non-invasive testing for HP infection, followed by eradication ('test and treat' strategy), is recommended for patients that are persistently dyspeptic and are under the age of 45, without alarming features. However, the effectiveness of HP 'test and treat' is low in populations with a low HP prevalence and in this situation empirical acid suppression is an equivalent option. Once a patient has failed a 4- to 8-week trial of proton-pump inhibitor therapy (in a geographical area with a low prevalence of HP) or failed to respond to HP eradication (in a HP-endemic region), upper endoscopy is indicated. In countries with a higher incidence of HP infection, 'test and treat' leads to a reduced endoscopic workload and is cost-effective (28). Thus, screening for HP identification seems to be a suitable serological test to non-invasively diagnose pre-malignant gastric lesions, given the central role of this bacterium in gastric carcinogenesis.

Non-invasive tests for the diagnosis of HP infection include: the ${ }^{13} \mathrm{C}$ urea breath test, stool antigen tests (with polyclonal or monoclonal antibodies) and immunological tests (laboratoryand office-based tests and tests on saliva and urine). The urea breath procedure is an accurate, practical and readily available test, with a sensitivity of $94 \%$ and a specificity of $95 \%$. The stool antigen test has a sensitivity and specificity of 91 and $93 \%$, respectively; the sensitivity decreases to $69 \%$ after the sample is left standing for 2-3 days at room temperature. Serology is a widely available and low-cost non-invasive test; however, the diagnostic accuracy is low (80-84\%).

Prior treatment with proton-pump inhibitors can result in false-negative, invasive and non-invasive diagnostic tests. Therefore, these drugs should be stopped for at least 2 weeks prior to testing. However, this advice does not apply to serology. Nonetheless, while serologic office-based tests are extremely convenient, they are not accurate and are currently not recommended for the detection of HP infection. Serology should be considered as a diagnostic test only in the case of false-negative results by other methods, such as in patients with bleeding ulcers, gastric atrophy, MALT lymphoma, or in those being treated with proton-pump inhibitors and antibiotics.

The detection of specific HP antibodies in urine and saliva has no current role in patient management but may be helpful for epidemiological studies. Since HP virulence factors differ and 
host genetic factors may influence disease outcome, neither the routine detection of HP pathogenic factors nor the assessment of host genetic polymorphisms is currently recommended. The rapid urease test can detect the presence of HP within $1 \mathrm{~h}$, with a satisfactory accuracy (90\%). False-negative results can occur in patients taking anti-secretory drugs. In patients presenting for endoscopy without pre-treatment, a positive rapid urease test is sufficient to initiate treatment. Non-invasive tests should be employed for the confirmation of eradication, except in cases in which repeat endoscopy is indicated, such as in patients with gastric ulcers. When the urea breath test is not available, a stool test, which is less accurate, may be used, preferably analyzed with monoclonal antibodies. Confirmation of HP eradication should be assessed at least 4 weeks following treatment (29).

Pepsinogen and gastrin levels, in combination with HP serology, are useful to establish with high sensitivity and specificity the presence of gastric pre-malignant lesions, particularly atrophic gastritis. Serological tests for pepsinogens I and II and for gastrin provide valuable information on the status of the gastric mucosa. As is well known, pepsinogen I is produced by mucosal neck cells in the fundic glands, whereas pepsinogen II is produced throughout the entire stomach by mucosal neck cells and by pyloric and Brunner's glands. Gastric inflammation causes an increased release of both pepsinogens into the bloodstream, with a greater increase in pepsinogen II than in pepsinogen I production. Atrophic gastritis causes a decrease in the production of both pepsinogens which is more pronounced for pepsinogen I than for pepsinogen II. As a result of these changes, chronic gastritis is associated with a reduced pepsinogen I/II ratio, which decreases even further when atrophic gastritis occurs. In addition, gastrin is synthesized and secreted from antral G-cells. HP gastritis tends to raise gastrin serum levels, possibly due to hyperplasia of the antral G-cells and to an acid-suppressive effect of chronic gastritis when the corpus mucosa is involved. The increased production of gastrin also occurs in patients with atrophic gastritis of the corpus, in response to reduced acid secretion. By contrast, gastrin levels decrease in patients with antral-predominant atrophic gastritis (30).

Pre-malignant gastric lesions are often diagnosed by histological examination of random biopsy samples. At present, the Sydney system is generally used, both in clinical practice and in research, to grade gastritis. In this classification system, several features of inflammation, atrophy and intestinal metaplasia are separately assessed and then graded. Thus, atrophic gastritis is defined as loss of glandular structures of the gastric mucosa and intestinal metaplasia as the replacement of gastric columnar epithelial cells by cells with an intestinal morphology. Intestinal metaplasia possibly results from the differentiation of gastric stem cells into cells with a small intestinal phenotype or colonic components. It is characterized by the presence of intestinal-type, mucin-containing goblet cells, Paneth cells and absorptive cells. Gastric dysplasia (formerly non-invasive neoplasia; synonym, intraepithelial neoplasia) is characterized by epithelial cells that vary in size, shape and orientation, with nuclear enlargement and atypia as well as the distortion of the normal glandular arrangement (12).

It should be emphasized that differences exist between Japanese and Western gastrointestinal pathologists with respect to the classification of gastric dysplasia and cancer. Japanese pathologists diagnose cancer based on cellular and structural abnormalities, whereas Western pathologists focus on the presence of tissue invasion as a prerequisite for a diagnosis of cancer (31). In the year 2000, the unified Padua classification was proposed, which divides dysplasia and adenocarcinoma into 5 categories. The Vienna classification further distinguishes the categories of low-and high-grade dysplasia and was revised to improve the correlation with clinical management $(32,33)$.

Recently, an international group of gastroenterologists and pathologists [the Operative Link for Gastritis Assessment (OLGA)], proposed a system for reporting gastritis in terms of stage (the OLGA staging system), which arranges the histological phenotypes of gastritis along a scale of progressively increasing GC risk, from the lowest (OLGA stage 0) to the highest (OLGA stage IV). This staging framework is borrowed from the oncology vocabulary and it applies to a gastritis histology-based reporting format that was successfully adopted for chronic hepatitis. Just as a given number of portal tracts is required for the accurate staging of hepatitis, a well-defined biopsy sampling protocol (as recommended by the Sydney system) is a 'minimum requirement' for the reliable staging of gastritis, which is carried out by combining the extent of atrophy (scored histologically) with the topographical location of the tumor (as seen on the mapping protocol) (34).

Although the image quality of standard endoscopy has improved over the last several decades, the findings at conventional endoscopy often do not correlate with the histological diagnoses of gastric pre-malignant lesions. This results from an unsatisfactory visualization of structure, color and vascularity by conventional techniques, as all these features play a role in the adequate distinction of pre-malignant and early $\mathrm{GC}$ lesions. Consequently, several alternative and supplementary strategies have been developed to overcome the limitations of standard endoscopic imaging, such as magnification endoscopy in patients with pre-malignant gastric lesions or early GC.

Methylene blue staining is based on the absorptive capability of cells and is used to demonstrate intestinal metaplasia, whereas Indigo carmine enhances the architectural changes in neoplastic lesions. Chromoendoscopy is an important approach to the detection of pre-malignant gastric lesions and to the identification of small foci of early gastric carcinoma not visible with white light gastroscopy (35). A number of techniques that use the specific spectral and absorptive features of light have been developed, including narrow-band imaging, autofluorescence and hemoglobin enhancement. Narrow-band imaging has been evaluated in combination with magnification endoscopy. This technique uses narrow filtered bands in the excitation light to improve imaging of the superficial capillary network and of the surface contrast within the mucosa. In autofluorescence endoscopy, the tissue is exposed to light of shorter wavelengths, typically blue light, with the subsequent emission of light by endogenous fluorophores. Finally, the super-addition of a pseudocolor image based on the mucosal hemoglobin content (hemoglobin enhancement) can be used to facilitate the delineation of lesions (30).

\section{Screening and surveillance}

Since the incidence of GC is geographically highly variable, the development of uniform worldwide screening strategies seems 
inappropriate, given that, in screening, the burden for patients as well as the costs and the restricted availability of specific therapeutic interventions need to be taken into consideration. A high individual risk justifies invasive investigation by endoscopy, whereas a more conservative approach is appropriate and ethically acceptable in individuals in low-risk regions. The initial selection of subjects for screening in low-incidence countries should possibly be based uniquely on epidemiological factors, such as age, country of birth and socioeconomic class, all of which are risk factors for HP infection. Initial screening would consist of non-invasive tests (36).

A serological diagnosis of atrophic gastritis should be followed by endoscopy, with histological confirmation of the diagnosis. However, in high-incidence countries, serologic and endoscopic screening could be offered to the general population, as is common practice in Japan. Indeed, in Japan, mortality rates have declined, which may be ascribed to the introduction of photofluorography. Screening may have also contributed to the persistently high incidence rates reported by the Japanese (37). Apart from screening, endoscopic surveillance of patients with pre-malignant lesions is an important approach to reduce GC morbidity and mortality. Surprisingly, clear guidelines are not available for the surveillance of patients with atrophic gastritis, intestinal metaplasia, or gastric dysplasia, even more so since the guidelines for surveillance of other gastrointestinal premalignant conditions have been widely developed, for instance for Barrett's esophagus or colonic adenomas. Thus, the 'screen and treat' strategy for HP infection should be considered an effective strategy for GC prevention only in communities with a high incidence of GC (38).

The diagnosis of gastric MALT lymphoma is based on the histopathological evaluation of gastric biopsies and on immunohistochemistry. Gastric MALT lymphoma is a B-cell, low-grade, typically $\mathrm{CD}_{1}{ }^{+}, \mathrm{CD} 20^{+}$, usually $\mathrm{CD}^{-}$, always CD10 and CD23- ${ }^{-}$HL. Based on the close association between MALT lymphoma and HP, the identification of this infection is highly recommended.

MALT lymphomas behave clinically as indolent NHLs, with a long disease-free and overall survival. Their good prognosis is likely related to their tendency to remain localized for long periods of time and to the low frequency of transformation into aggressive NHL. Often, only vague dyspeptic symptoms characterize gastric MALT lymphoma and B symptoms are extremely rare, so that the diagnosis is often incidental. In other cases, it may first be detected as a complication of the gastric lesion, such as gastrointestinal bleeding or perforation. Persistent vomiting and weight loss are other possible presenting symptoms (39).

In approximately $50 \%$ of cases, gastric MALT lymphomas disseminate within the gastrointestinal tract. The most common non-gastric primary sites are the salivary glands and ocular adnexa (25\% of cases each), lung (14\%) and skin (12\%). Extra-gastric MALT lymphomas are significantly more diffuse than gastric MALT lymphomas (50 vs. 25\%), thus reflecting a particular homing pattern of lymphocytes generated within a MALT environment, a possible difference between gastrointestinal and non-gastrointestinal MALT.

In terms of genetic aberrations, 2 subgroups with a higher risk of dissemination can be identified: i) $\mathrm{t}(11 ; 18)(\mathrm{q} 21 ; \mathrm{q} 21)$ gastric MALT lymphomas and ii) extra-gastric MALT lymphomas with trisomy 18 (40). HP-negative gastric MALT lymphoma accounts for 5-10\% of all gastric MALT lymphomas. Clinically, HP-negative tumors are located more frequently in the proximal portion of the stomach and are less frequently of the superficial type. HP-negative tumors may include those related to autoimmune gastritis, which predominantly involves the corpus, whereas antrum-predominant gastritis associated with HP-negative gastric MALT lymphoma frequently displays $\mathrm{t}(11 ; 18)(\mathrm{q} 21 ; \mathrm{q} 21)$ and Bcl-10 nuclear expression. However, HP-negative gastric MALT lymphoma has a favorable long-term outcome, comparable to that of HP-positive lymphoma (41). The presence of genetic alterations in cells of MALT lymphoma, such as trisomy 3, API2-MALT1 translocation, p53 mutation and p16 deletion, characterizes neoplastic B cells with aggressive behavior, causing the so-called lympho-epithelial lesions. These are a pathognomonic sign of lymphoma, with the invasion and disruption of gastric glands.

At endoscopic observation, MALT lymphoma may present with different macroscopic features, ranging from a normal-appearing gastric mucosa to an ulcerative or vegetant mass, clearly suggesting a malignancy. In addition to routine histological analysis and immunohistochemistry, FISH analysis (or PCR) for the detection of $\mathrm{t}(11 ; 18)$ may be useful in identifying patients unresponsive to antibiotic therapy. Rarely, patients may have elevated lactate dehydrogenase or $\beta 2$-microglobulin levels. A majority of patients have no abnormal findings on physical examination. The endoscopic appearance of gastric low-grade lymphoma often mimics that of benign diseases, such as chronic gastritis or a peptic ulcer. The histological evaluation of subsequent biopsies remains an essential follow-up procedure in MALT lymphoma. Unfortunately, the interpretation of a lymphoid infiltrate in post-treatment gastric biopsies can be very difficult and there are no uniform criteria for the definition of histological remission. A preliminary breath test or stool antigen test should be performed at least 4 weeks following antibiotic treatment to establish whether or not HP has been eradicated. Moreover, a strict endoscopic follow-up is recommended, with multiple biopsies taken 2-3 months after treatment and subsequently at least twice per year for 2 years, to monitor the histological regression of the lymphoma. Gastric MALT lymphomas have a low tendency to distant spreading and to histological transformation. Long-term careful endoscopic control and systemic (blood counts and minimal adequate radiological or ultrasound examinations) follow-up once a year are recommended for all patients. Indeed, the risk of gastric adenocarcinoma among patients diagnosed with gastric MALT lymphoma is reportedly 6-fold higher than in the general population $(42,43)$.

Gastric polyps are associated with an elevated frequency of pre-cancerous alterations of the gastric mucosa and, consequently, with an elevated risk of synchronous or metachronous cancer. They are often asymptomatic. Esophagogastroduodenoscopy is the gold standard for their diagnosis. With the expanding indications for this examination, polyps are more frequently detected; currently, they are identified in approximately 5\% of upper gastrointestinal tract endoscopies, mostly as incidental findings (44). One of the main recommendations of the British Society of Gastroenterology is that a biopsy sample should be obtained from all gastric polyps detected at endoscopy. Histopathological assessment is required to establish the diagnosis and to identify the dysplastic 
Table I. Clinical features and management of gastric polyps.

\begin{tabular}{|c|c|c|c|c|c|}
\hline Polyp type & $\begin{array}{l}\text { Prevalence } \\
\text { (frequency } \\
\text { relative to } \\
\text { other polyps) }\end{array}$ & $\begin{array}{l}\text { Usual number, } \\
\text { size and site }\end{array}$ & $\begin{array}{c}\text { Malignant } \\
\text { potential }\end{array}$ & $\begin{array}{c}\text { Pathological } \\
\text { features of } \\
\text { background } \\
\text { gastric mucosa }\end{array}$ & Management \\
\hline Fundic & $13-77 \%$ & $\begin{array}{l}\text { Multiple; } \\
1-5 \mathrm{~mm} ; \\
\text { upper and } \\
\text { lower body }\end{array}$ & Low & $\begin{array}{l}\text { Associated with PPI } \\
\text { use; may regress after } \\
\text { interruption of PPI. } \\
\text { No dysplasia. } \\
\text { Consider FAP }\end{array}$ & $\begin{array}{l}\text { Biopsy to confirm } \\
\text { histological nature. } \\
\text { No follow-up }\end{array}$ \\
\hline Hyperplastic & $18-70 \%$ & $\begin{array}{l}\text { Single or } \\
\text { multiple; } \\
0.5-15 \mathrm{~mm} \text {; } \\
\text { antrum or } \\
\text { lower body }\end{array}$ & Low & $\begin{array}{l}\text { HP associated } \\
\text { gastritis }(25 \%) \text {; } \\
\text { may regress after } \\
\text { eradication therapy. } \\
\text { Dysplasia is } \\
\text { rare }(<3 \%)\end{array}$ & $\begin{array}{l}\text { Remove polyp } \\
\text { if dysplastic or } \\
\text { symptomatic. } \\
\text { Eradicate HP } \\
\text { Repeat gastroscopy } \\
\text { after } 1 \text { year }\end{array}$ \\
\hline Adenomatous & $\begin{array}{l}0.50-3.75 \% \\
\text { (in Western } \\
\text { countries); } \\
9-27 \% \\
\text { (in Eastern } \\
\text { countries) }\end{array}$ & $\begin{array}{l}\text { Usually } \\
\text { single; } \\
<20 \mathrm{~mm} \text {; } \\
\text { antrum }\end{array}$ & High & $\begin{array}{l}\text { Atrophic gastritis } \\
\text { with intestinal } \\
\text { metaplasia. } \\
\text { Malignant foci } \\
\text { may coexist }\end{array}$ & $\begin{array}{l}\text { Polypectomy. } \\
\text { Repeat gastroscopy } \\
\text { after } 1 \text { year }\end{array}$ \\
\hline
\end{tabular}

HP, Helicobacter pylori; PPI, proton-pump inhibitors; FAP, familial adenomatous polyposis.

foci. All patients who have hyperplastic or adenomatous polyps at esophagogastroduodenoscopy should be evaluated for HP infection, in that its eradication is associated with the regression of approximately $80 \%$ of hyperplastic polyps whereas the advantage of HP eradication in adenomas is less clear. In the surveillance of gastric polyps, gastroscopy should be repeated after one year for all polyps with dysplasia that have not been removed, after 6 months for adenomatous polyps with high-grade dysplasia and after one year following complete polypectomy for all other high-risk polyps (Table I).

The correct management of hereditary cancer syndromes requires genetic counseling, information on the family pedigree going back 3 generations, histopathological confirmation of gastric carcinoma and informed consent. The criteria for screening GC families for HDGC require confirmed diffuse GC in a minimum of 2 first- or second-degree relatives, provided one is under 50 years of age, or 3 confirmed cases in family members of any age. Approximately $30 \%$ of families meeting these original criteria carry a predisposing CDH1 mutation (E-cadherin). This autosomal dominant mutation is seen in at least one fourth of the cases of HDGC, with a relatively high penetrance and a 70-80\% lifetime risk of GC occurrence (45).

Patients with HDGC typically present with diffuse-type GC with signet ring cells and, at later stages, linitis plastica. Advanced hereditary and sporadic diffuse forms of GC are indistinguishable. Anatomical mapping of complete gastrectomy specimens has shown that early-stage HDGC is characterized by the presence of multiple foci of stage T1a, signet ring cells confined to the superficial lamina propria and the absence of nodal metastases. These foci are submucosal and not readily identified by gastroscopy. The majority of foci appear relatively indolent, with scanty mitoses, although they can rapidly progress into advanced disease. Therefore, in early gastric lesions it is important for pathologists to recognize a phenotype consisting of patchy intramucosal signet ring cells often associated with pagetoid spread. Even advanced-stage diffuse GC can be missed at gastroscopy as it can infiltrate below an intact epithelium. Improved endoscopic techniques that can detect submucosal foci, coupled with histological or immunological markers, are urgently required.

Despite these limitations, surveillance endoscopy should be carried out annually using white light, high-definition endoscopy: 1i) for carriers of mutation who decline prophylactic surgery; ii) for patients younger than the age at which prophylactic surgery is recommended (approximately 20 years); and iii) prior to prophylactic surgery in newly diagnosed carriers (46).

\section{Prevention and treatment}

Since symptoms are often absent or non-specific, GC is frequently diagnosed at an advanced stage, when therapeutic options are limited and prognosis is poor. Consequently, the overall 5-year survival of these patients is $<20 \%$ (1).

As previously stated, HP infection is considered an important initial step in gastric carcinogenesis, conferring a 6-fold higher risk. The chronic inflammation of the gastric mucosa associated with HP infection slowly progresses through the aforementioned pre-malignant stages to gastric adenocarcinoma. Since it has been estimated that $50 \%$ of the world population is infected 


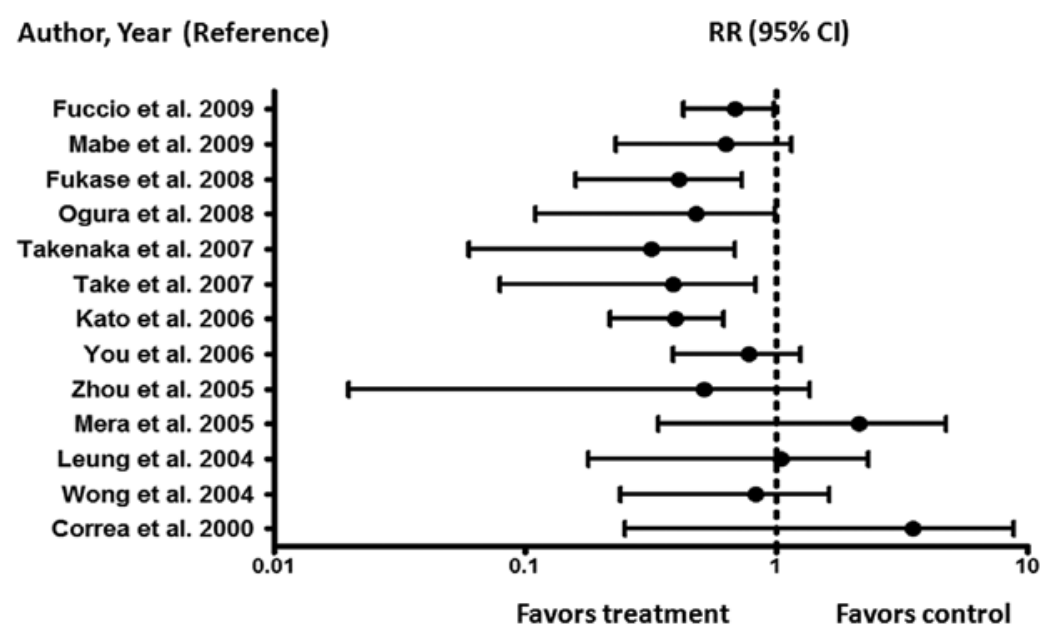

Figure 4. Effect of Helicobacter pylori eradication on the risk of gastric cancer: meta-analysis of clinical trials.

with the bacterium, its eradication seems a logical step in the therapy of chronic gastritis and in the prevention of GC. The aim of HP eradication therapy is either to restore the inflamed mucosa to its normal healthy state or to prevent further progression of advanced chronic lesions (atrophic gastritis and intestinal metaplasia). Accordingly, it is better to administer therapy prior to the onset of these pre-neoplastic mucosal abnormalities (47). It should be emphasized that HP eradication from the residual gastric mucosa after the endoscopic treatment of early GC may also result in an inhibitory effect on the occurrence of metachronous GC (48). Conversely, several meta-analyses have shown that the progression of atrophic gastritis and intestinal metaplasia to GC can indeed occur following HP eradication, suggesting that other factors contribute to the progression of pre-neoplastic lesions (48). For example, genetic and epigenetic alterations may reach a point of no return, even after elimination of the triggering carcinogen. Thus, the actual role of HP eradication in the prevention of GC is still a matter of wide debate. Fig. 4 summarizes the meta-analyses of randomized controlled trials and non-randomized studies aimed at assessing whether HP eradication is indeed an effective preventive strategy to reduce the risk of developing GC (9,48,50-61).

For low-grade MALT lymphomas confined to the gastric wall or perigastric lymph nodes (stages I or II), a meta-analysis of more than 30 studies (8) yielded an overall remission rate of $78 \%$ (95\% confidence interval: $75-80 \%)$. According to this same report, the overall rate of lymphoma recurrence was only $2 \%$ per year. Therefore, HP eradication should be the first-choice treatment in patients with HP-positive MALT lymphoma $(42,62)$. Candidates for anti-HP therapy are also patients with localized, mucosal, or submucosal, non-bulky, flat disease who do not have metastases, lymphadenopathy, or frank diffuse large B-cell lymphomas. It is estimated that $<10 \%$ of patients with gastric lymphoma are candidates for antibiotic treatment as first-line therapy, although recent data suggested that HP-negative patients will also benefit from this therapeutic approach $(63,64)$.

In patients who have not responded to eradication therapy, surgical, radiation and systemic therapies (with chemotherapeutic agents and/or anti-CD20 monoclonal antibodies) should be administered, taking into account the stage of the disease. Since the majority of patients are responsive to eradication therapy, the timing of a new biopsy for response assessment is crucial to avoid an inappropriate conclusion of failure. Complete resolution of the lymphoid infiltrate usually takes from several months to as long as 2 years. However, molecular evidence of clonality, as determined by $\mathrm{IgH}$ gene rearrangement studies, may persist for years after morphological remission but without affecting patient outcome (42).

The $\mathrm{t}(11 ; 18)$ translocation involves fusion of the $\mathrm{N}$-terminus of API2 (apoptosis inhibitor-2) on chromosome 11 and the C-terminus of MALT1 on chromosome 18. It is the most common cytogenetic abnormality found in MALT lymphomas of the gastrointestinal tract, occurring in up to $25 \%$ of gastric MALT lymphomas. This translocation correlates with resistance to antibiotic therapy and with disseminated rather than stage I disease. Although molecular studies are not routinely performed, a search for $\mathrm{t}(11 ; 18)(\mathrm{q} 21 ; \mathrm{q} 21)$ translocation should be considered in order to determine prognosis and therapy for patients refractory to conservative treatment (65).

Generally, the eradication of HP infection is recommended in the following high-risk situations: i) patients with gastric MALT lymphoma; ii) patients with atrophic gastritis; iii) first-degree relatives of patients with gastric intestinal or diffuse type of cancer; iv) patients with unexplained iron-deficiency anemia; v) patients with gastroesophageal reflux disease requiring long-term acid-suppression therapy, due to the potential of proton-pump inhibitors to induce, in the presence of HP, atrophic gastritis, with a subsequent risk of developing GC; vi) patients with early GC treated by endoscopic mucosal resection, as it can remove lesions posing a minimal mortality risk, but likely to have higher rates of recurrence and incomplete resections; and vii) patients with partial gastrectomy for GC $(28,29)$.

Triple therapy (proton-pump inhibitors + amoxicillin + clarithromycin) is the first-line regimen for the eradication of HP. Metronidazole treatment is the recommended first-choice treatment in association with proton-pump inhibitors and amoxicillin in individuals with clarithromycin resistance (66), which account for $<15-20 \%$ of the general population (67). 
Quadruple therapy (proton-pump inhibitors + bismuth subcitrate potassium + metronidazole + tetracycline) remains the best second-choice treatment. Recent studies have indicated that quadruple therapy induces a higher eradication rate, whereas its safety and tolerability are similar to those of triple therapy (68). If second-line eradication therapy fails or if there is resistance to metronidazole, an event that occurs in approximately $40 \%$ of cases (67), a proton-pump inhibitor + amoxicillin + levofloxacin is recommended. This combination is expected to be effective, with a relatively low rate of adverse reactions, although the more frequent use of the new quinolones seems to result in increased resistance. Rifabutin-based triple therapy has also been reported to be an effective salvage therapy (66).

Proton-pump inhibitors are crucial for HP eradication. They increase the stability of acid-labile antibiotics and, by increasing the drug concentration in the gastric juice, improve the sensitivity of HP. It is even possible that proton-pump inhibitors have intrinsic, albeit modest, antimicrobial properties. The metabolism of proton-pump inhibitors depends on hepatic cytochrome P450 enzymes, particularly the CYP2C19 genotype, which displays 3 polymorphisms that can interfere in drug metabolism and thus the pharmacodynamics of proton-pump inhibitors. An increased dose of esomeprazole (40 mg twice daily) in triple therapy may therefore result in a better HP eradication rate than achieved with omeprazole-based therapy for the wild-type, homozygous genotype of CYP2C19. The choice of proton-pump inhibitors and their respective dosage together with the evaluation of CYP2C19 genotyping may be a more rational approach to obtain the highest HP eradication rates in a clinical setting. Currently, however, this approach is hardly applicable due to the elevated costs and the limited availability of genotyping (69).

Sequential therapy has been proposed as an alternative to standard triple therapy for the eradication of HP. The primary goal of this regimen is to overcome clarithromycin resistance. Hypothetically, during the first 5 days of therapy amoxicillin would weaken the bacterial cell wall, thus preventing the formation of the channels which block clarithromycin from entering the bacterium and thereby causing resistance to this antibiotic. In the second phase, clarithromycin and nitroimidazole are added for 5 additional days. The proton-pump inhibitor is continued throughout treatment. However, there are currently insufficient data to recommend sequential therapy as a first-line choice for HP therapy. A longer treatment duration, namely 14 instead of 7 days (66), may increase eradication rates; however, this approach remains controversial.

Non-invasive tests should be employed to confirm eradication, except when a new endoscopy is indicated, such as in patients with gastric ulcers. During follow-up after eradication therapy, the urea breath test is the best option, based on its high sensitivity and specificity. As noted above, the stool antigen test is less accurate than the urea breath test; however, it is an acceptable alternative if the latter is not available. Confirmation of HP eradication should be performed at least 4 weeks after treatment (70). Nonetheless, $1 \%$ of patients still develop GC despite successful HP eradication; furthermore, a tumor may develop several years following HP eradication. Thus, it should be kept in mind that GC, similar to other tumors, is a multifactorial disease and removing one factor does not prevent all of these malignancies (49).
In consideration of the increased incidence of treatment failure and antibiotic resistance, the development of a prophylactic or therapeutic vaccine against HP seems a desirable alternative, which may improve the rate of eradication success obtained with standard regimens or reduce the bacterial density in the gastric mucosa. Although vaccination studies in animal models have yielded promising results, experiments in human volunteers have revealed problems such as 'post-immunization gastritis'. Moreover, responses to vaccine antigens have been comparatively poor, possibly since HP colonizes the gastric mucosa without crossing the epithelium, thus making the bacterium inaccessible to many immune effector mechanisms. While HP stimulates both innate and acquired immune responses, it nonetheless persists by exploiting a mechanism of reduced identification based on the generation of different epitopes through point mutations and recombinations. It also evades and manipulates the immune system by sequestering itself in the gut lumen, where the immune system is less efficient, while also mimicking host antigens and downregulating the activation of immune cells (71).

New directions for active immunization include the use of DNA, living vectors, microspheres, new vaccination schedules and different routes of administration (oral, intranasal, rectal and intramuscular). Many of these new approaches are currently being evaluated (72). In a recent study, a multiepitope DNA-prime/peptide-boost immunization strategy was proposed, using informatics tools in a mouse model of GC induced by chronic HP infection. The multi-epitope vaccine was administered intranasally and induced a broad immune response, as determined by interferon- $\gamma$ production in ELISpot assays. These results suggest that the development of an epitope-based mucosal vaccine may be beneficial in eradicating HP and in reducing the burden of associated GC in humans (73).

As previously stated, hyperplastic polyp formation is strongly associated with HP-positive gastritis. Up to $80 \%$ of hyperplastic polyps regress after HP eradication and therefore do not require endoscopic removal. Obviously, eradication therapy is not indicated for the other types of gastric polyps as they are not associated with HP infection. Conversely, all gastric adenomatous polyps and all gastric polyps with dysplastic foci, as well as symptomatic polyps, should be removed. Endoscopic mucosal resection has become the standard of care for the removal of gastric large, flat and sessile polyps and early cancer. Recently, endoscopic submucosal dissection was introduced in Japan as an alternative technique allowing the 'en bloc' resection of large lesions. The indications for endoscopic mucosal resection are expanding and many Western endoscopists are adopting the technique (74).

The most important hereditary GC is HDGC syndrome, which is associated with CDH1 germline mutations. Prophylactic gastrectomy should be considered only when these mutations occur, in that they imply a $>80 \%$ lifetime risk of developing GC. Other inherited cancer predispositions are hereditary non-polyposis colorectal cancer and Li-Fraumeni and Peutz-Jeghers syndromes; in these cases, only endoscopic surveillance is recommended $(46,75)$.

The overexpression of the COX-2 gene in GC suggests its role in gastrointestinal carcinogenesis. The enzyme participates in several key cellular activities, including cell proliferation, 
apoptosis and angiogenesis. NSAIDs and aspirin are therefore potential agents for the chemoprevention of GC. The impact of HP infection on the relationship between the use of NSAIDs and GC remains unclear. HP infection is known to be associated with non-cardia GC. Thus, it is possible that NSAIDs inhibit the replication and proliferation of HP, thus neutralizing the increased COX-2 expression and elevated prostaglandin synthesis associated with HP infection while reducing the risk of GC (76).

A strong correlation can also be envisaged between HP infection and dietary factors in gastric carcinogenesis. In fact, HP gastritis enhances the growth of nitrose-producing bacteria, which catalyze the production of carcinogenic $\mathrm{N}$-nitrose compounds. In addition, HP infection is known to inhibit the gastric secretion of ascorbic acid, which is an important scavenger of $\mathrm{N}$-nitrose compounds and oxygen-free radicals. Moreover, salt-preserved foods and the dietary nitrite found in preserved meats are potentially carcinogenic. The intake of salted food may increase the risk of HP infection and act synergistically to promote the development of GC. Salt-induced mucosal damage may increase the possibility of persistent infection with HP (4). Given that GC is often associated with a poor prognosis, the main strategy for improving clinical outcome remains prevention.

The widespread introduction of refrigeration has further decreased the intake of chemically preserved foods and increased the consumption of fresh fruits and vegetables. A decline in the prevalence of HP infection may be due to improvements in sanitary and housing conditions, as well as the use of eradication therapy. In consideration of the multifactorial pathogenesis of GC, all modifiable risk factors, such as high salt and nitrite consumption, low fruit and vegetable intake, cigarette smoking and HP infection may represent targets for prevention (77).

\section{Conclusions}

Over the past decades, HP infection has been clearly correlated with gastric carcinogenesis. The strongest support for a link between HP infection and GC development has come from the preventive effect of HP eradication. At present and in the near future, the most important challenge is to significantly reduce mortality due to $\mathrm{GC}$, as the prognosis of patients with GC remains extremely poor. This can be achieved by the identification of higher-risk patients, such as those with atrophic gastritis, intestinal metaplasia, dysplasia of the stomach and, in rare cases, in those with hyperplastic gastric polyps or germline mutations in CDH1, responsible for HDGC. A number of recent studies on gastric pre-cancerous lesions have offered the promise of novel biomarkers allowing for the early detection of GC, while others have reported improvements in invasive and non-invasive diagnostic tests for pre-malignant stages of GC. In conclusion, recognition of the pre-malignant condition at an early stage is a major advantage as it allows endoscopic surveillance and, possibly, therapeutic mucosal resection. In addition, preventive cancer strategies, such as HP eradication with triple or quadruple treatment, the development of prophylactic or therapeutic vaccination against HP and perhaps chemoprevention in the form of NSAIDs and aspirin, against a background of lifestyle and dietary modifications, may be effective in 'at risk' populations.

\section{Acknowledgements}

This study was supported in part by grants from the Italian Association for Cancer Research (AIRC, Milan, Italy), the Italian Foundation 'Cassa di Risparmio di Puglia' (Bari, Italy) and the strategic project 'Biotecnoter' of the Apulia Region (Bari, Italy).

\section{References}

1. Jemal A, Bray F, Center MM, Ferlay J, Ward E and Forman D: Global cancer statistics. CA Cancer J Clin 61: 69-90, 2011.

2. Kim SS, Ruiz VE, Carroll JD and Moss SF: Helicobacter pylori in the pathogenesis of gastric cancer and gastric lymphoma. Cancer Lett 305: 228-238, 2011.

3. Suerbaum S and Michetti P: Helicobacter pylori infection. N Engl J Med 347: 1175-1186, 2002.

4. Fuccio L, Eusebi LH and Bazzoli F: Gastric cancer, Helicobacter pylori infection and other risk factors. World J Gastrointest Oncol 2: 342-347, 2010.

5. Conteduca V, Sansonno D, Ingravallo G, et al: Barrett's esophagus and esophageal cancer: an overview. Int J Oncol 41: 414-424, 2012.

6. Crew KD and Neugut AI: Epidemiology of gastric cancer. World J Gastroenterol 12: 354-362, 2006.

7. Polk DB and Peek RM Jr: Helicobacter pylori: gastric cancer and beyond. Nat Rev Cancer 10: 403-414, 2010.

8. Zullo A, Hassan C, Cristofari F, et al: Effects of Helicobacter pylori eradication on early stage gastric mucosa-associated lymphoid tissue lymphoma. Clin Gastroenterol Hepatol 8: 105-110, 2010.

9. Fuccio L, Zagari RM, Eusebi LH, et al: Meta-analysis: can Helicobacter pylori eradication treatment reduce the risk for gastric cancer? Ann Intern Med 151: 121-128, 2009.

10. Yaghoobi M, Bijarchi R and Narod SA: Family history and the risk of gastric cancer. Br J Cancer 102: 237-242, 2010.

11. IARC Working Group: Schistosomes, liver flukes and Helicobacter pylori. IARC Working Group on the Evaluation of Carcinogenic Risks to Humans. Lyon, 7-14 June 1994. IARC Monogr Eval Carcinog Risks Hum 61: 1-241, 1994.

12. De Vries AC, Haringsma $\mathbf{J}$ and Kuipers EJ: The detection, surveillance and treatment of premalignant gastric lesions related to Helicobacter pylori infection. Helicobacter 12: 1-15, 2007.

13. Correa P: A human model of gastric carcinogenesis. Cancer Res 48: 3554-3560, 1998.

14. Watanabe T, Tada M, Nagai H, Sasaki S and Nakao M: Helicobacter pylori infection induces gastric cancer in mongolian gerbils. Gastroenterology 115: 642-648, 1998.

15. Correa $\mathrm{P}$ and Houghton J: Carcinogenesis of Helicobacter pylori. Gastroenterology 133: 659-672, 2007.

16. Hatakeyama M: Helicobacter pylori CagA-a bacterial intruder conspiring gastric carcinogenesis. Int J Cancer 119: 1217-1223, 2006.

17. Konturek PC, Konturek SJ and Brzozowski T: Helicobacter pylori infection in gastric cancerogenesis. J Physiol Pharmacol 60: 3-21, 2009.

18. Gianfagna F, De Feo E, van Duijn CM, Ricciardi G and Boccia S: A systematic review of meta-analyses on gene polymorphisms and gastric cancer risk. Curr Genomics 9: 361-374, 2008.

19. González CA, Figueiredo C, Lic CB, et al: Helicobacter pylori cagA and vacA genotypes as predictors of progression of gastric preneoplastic lesions: a long-term follow-up in a high-risk area in Spain. Am J Gastroenterol 106: 867-874, 2011.

20. Liu D, He Q and Liu C: Correlations among Helicobacter pylori infection and the expression of cyclooxygenase- 2 and vascular endothelial growth factor in gastric mucosa with intestinal metaplasia or dysplasia. J Gastroenterol Hepatol 25: 795-799, 2010.

21. Hold GL, Rabkin CS, Chow WH, et al: A functional polymorphism of toll-like receptor 4 gene increases risk of gastric carcinoma and its precursors. Gastroenterology 132: 905-912, 2007.

22. Nobili S, Bruno L, Landini I, et al: Genomic and genetic alterations influence the progression of gastric cancer. World $\mathrm{J}$ Gastroenterol 17: 290-299, 2011. 
23. Lu XX, Yu JL, Ying LS, et al: Stepwise cumulation of RUNX3 methylation mediated by Helicobacter pylori infection contributes to gastric carcinoma progression. Cancer 118: 5507-5517, 2012.

24. Rocco A, Compare D and Nardone G: Cancer stem cell hypothesis and gastric carcinogenesis: Experimental evidence and unsolved questions. World J Gastrointest Oncol 4: 54-59, 2012.

25. Suarez F, Lortholary O, Hermine O, et al: Infection-associated lymphomas derived from marginal zone B cells: a model of antigen-driven lymphoproliferation. Blood 107: 3034-3044, 2006.

26. Carmack SW, Genta RM, Graham DY and Lauwers GY: Management of gastric polyps: a pathology-based guide for gastroenterologists. Nat Rev Gastroenterol Hepatol 6: 331-341, 2009.

27. Kelly PJ and Lauwers GY: Clinical guidelines: Consensus for the management of patients with gastric polyps. Nat Rev Gastroenterol Hepatol 8: 7-8, 2011.

28. Malfertheiner P, Megraud F, O'Morain C, et al: Current concepts in the management of Helicobacter pylori infection: the Maastricht III Consensus Report. Gut 56: 772-781, 2007.

29. Malfertheiner P, Megraud F, O'Morain CA, et al: Management of Helicobacter pylori infection - the Maastricht IV/ Florence Consensus Report. Gut 61: 646-664, 2012.

30. De Vries AC and Kuipers EJ: Epidemiology of premalignant gastric lesions: implications for the development of screening and surveillance strategies. Helicobacter 12: 22-31, 2007.

31. Schlemper RJ, Itabashi M, Kato Y, et al: Differences in diagnostic criteria for gastric carcinoma between Japanese and western pathologists. Lancet 349: 1725-1729, 1997.

32. Schlemper RJ, Riddell RH, Kato Y, et al: The Vienna classification of gastrointestinal epithelial neoplasia. Gut 47: 251-255, 2000.

33. Dixon MF: Gastrointestinal epithelial neoplasia: Vienna revisited. Gut 51: 130-131, 2002.

34. Rugge M, Pennelli G, Pilozzi E, et al: Gastritis: the histology report. Dig Liver Dis 43 (Suppl 4): S373-S384, 2011.

35. Ohnita $\mathrm{K}$, Isomoto $\mathrm{H}$, Shikuwa $\mathrm{S}$, et al: Magnifying chromoendoscopic findings of early gastric cancer and gastric adenoma. Dig Dis Sci 56: 2715-2722, 2011

36. Yeh JM, Hur C, Kuntz KM, Ezzati M and Goldie SJ: Costeffectiveness of treatment and endoscopic surveillance of precancerous lesions to prevent gastric cancer. Cancer 116 : 2941-2953, 2010

37. Hamashima C, Shibuya D, Yamazaki H, et al: The Japanese guidelines for gastric cancer screening. Jpn J Clin Oncol 38 259-267, 2008

38. Fock KM, Katelaris P, Sugano K, et al: Second Asia-Pacific consensus guidelines for Helicobacter pylori infection. J Gastroenterol Hepatol 24: 1587-1600, 2009.

39. Zullo A, Hassan C, Cristofari F, Perri F and Morini S: Gastric low-grade mucosal-associated lymphoid tissue-lymphoma: Helicobacter pylori and beyond. World J Gastrointest Oncol 2 : 181-186, 2010.

40. Oh SY, Kim WS, Kim JS, et al: Multiple mucosa-associated lymphoid tissue organs involving marginal zone B cell lymphoma: organ-specific relationships and the prognostic factors. Consortium for improving survival of lymphoma study. Int J Hematol 92: 510-517, 2010.

41. Shinagare AB, Ramaiya NH, O'Regan K, Jagannathan JP, Hornick JL and LaCasce AS: Helicobacter pylori-negative gastric mucosa-associated lymphoid tissue lymphoma. J Clin Oncol 29: 297-300,2011.

42. Zucca E and Dreyling M; ESMO Guidelines Working Group: Gastric marginal zone lymphoma of MALT type: ESMO Clinical Practice Guidelines for diagnosis, treatment and follow-up. Ann Oncol 21 (Suppl 5): v175-v176, 2010.

43. Ruskoné-Fourmestraux A, Fischbach W, Aleman BM, et al: EGILS consensus report. Gastric extranodal marginal zone B-cell lymphoma of MALT. Gut 60: 747-758, 2011.

44. Goddard AF, Badreldin R, Pritchard DM, et al; British Society of Gastroenterology: The management of gastric polyps. Gut 59: $1270-1276,2010$

45. Fitzgeral RC, Hardwick R, Huntsman D, et al: Hereditary diffuse gastric cancer: updated consensus guidelines for clinical management and directions for future research. J Med Genet 47: 436-444, 2010.

46. Guilford P, Humar B and Blair V: Hereditary diffuse gastric cancer: translation of $\mathrm{CDH} 1$ germline mutations into clinical practice. Gastric cancer 13: 1-10, 2010.
47. Bornschein J, Rokkas T, Selgrad M and Malfertheiner P: Helicobacter pylori and clinical aspects of gastric cancer. Helicobacter 14 (Suppl 1): 41-45, 2009.

48. Fukase K, Kato M, Kikuchi S, et al: Effect of eradication of Helicobacter pylori on incidence of metachronous gastric carcinoma after endoscopic resection of early gastric cancer: an open-label, randomised controlled trial. Lancet 372: 392-397, 2008.

49. Take S, Mizuno M, Ishiki K, et al: The long-term risk of gastric cancer after the successful eradication of Helicobacter pylori. J Gastroenterol 46: 318-324, 2011.

50. Kodama M, Murakami K, Okimoto T, et al: Helicobacter pylori eradication improves gastric atrophy and intestinal metaplasia in long-term observation. Digestion 85: 126-130, 2012.

51. Correa P, Fontham ET, Bravo JC, et al: Chemoprevention of gastric dysplasia: randomized trial of antioxidant supplements and anti-Helicobacter pylori therapy. J Natl Cancer Inst 92: 1881-1888, 2000

52. Wong BC, Lam SK, Wong WM, et al: Helicobacter pylori eradication to prevent gastric cancer in a high-risk region of China: a randomized controlled trial. JAMA 291: 187-194, 2004

53. Leung WK, Lin SR, Ching JY, et al: Factors predicting progression of gastric intestinal metaplasia: results of a randomised trial on Helicobacter pylori eradication. Gut 53: 1244-1249, 2004.

54. Mera R, Fontham ET, Bravo LE, et al: Long term follow up of patients treated for Helicobacter pylori infection. Gut 54: 1536-1540, 2005

55. Zhou LY, Lin SR, Ding SG, et al: The changing trends of the incidence of gastric cancer after Helicobacter pylori eradication in Shandong area. Chin J Dig Dis 6: 114-115, 2005.

56. You WC, Brown LM, Zhang L, et al: Randomized double-blind factorial trial of three treatments to reduce the prevalence of precancerous gastric lesions. J Natl Cancer Inst 98: 974-983, 2006.

57. Kato M, Asaka M, Nakamura T, et al: Helicobacter pylori eradication prevents the development of gastric cancer - results of a long-term retrospective study in Japan. Aliment Pharmacol Ther 24: 203-206, 2006

58. Take S, Mizuno M, Ishiki K, et al: Baseline gastric mucosal atrophy is a risk factor associated with the development of gastric cancer after Helicobacter pylori eradication therapy in patients with peptic ulcer diseases. J Gastroenterol 42 (Suppl 17): 21-27, 2007.

59. Takenaka R, Okada H, Kato J, et al: Helicobacter pylori eradication reduced the incidence of gastric cancer, especially of the intestinal type. Aliment Pharmacol Ther 25: 805-812, 2007.

60. Ogura K, Hirata Y, Yanai A, et al: The effect of Helicobacter pylori eradication on reducing the incidence of gastric cancer. J Clin Gastroenterol 42: 279-283, 2008.

61. Mabe K, Takahashi M, Oizumi H, et al: Does Helicobacter pylori eradication therapy for peptic ulcer prevent gastric cancer? World J Gastroenterol 15: 4290-4297, 2009.

62. Kuo SH, Yeh KH, Wu MS, et al: Helicobacter pylori eradication therapy is effective in the treatment of early-stage $\mathrm{H}$ pyloripositive gastric diffuse large B-cell lymphomas. Blood 119: 4838-4844, 2012

63. Park HS, Kim YJ, Yang WI, Suh CO and Lee YC: Treatment and outcome of localized Helicobacter pylori-negative low-grade gastric MALT lymphoma. World J Gastroenterol 16: 2158-2162, 2010.

64. Gill H, Chim CS, Au WY, et al: Non-gastric marginal zone B cell lymphoma: clinicopathologic features and treatment results. Ann Hematol 90: 1399-1407, 2011.

65. Owens SR and Smith LB: Molecular aspects of H. pylori-related MALT lymphoma. Patholog Res Int 2011: 193149, 2011.

66. O'Connor A, Gisbert JP, McNamara D and O'Morain C: Treatment of Helicobacter pylori infection 2010. Helicobacter 15: 46-52, 2010.

67. Graham DY and Fischbach L: Helicobacter pylori treatment in the era of increasing antibiotic resistance. Gut 59: 1143-1153, 2010.

68. Malfertheiner P, Bazzoli F, Delchier JC, et al: Helicobacter pylori eradication with a capsule containing bismuth subcitrate potassium, metronidazole and tetracycline given with omeprazole versus clarithromycin-based triple therapy: a randomised, open-label, non-inferiority, phase 3 trial. Lancet 377: 905-913, 2011.

69. Kang JM, Kim N, Lee DH, et al: Effect of the CYP2C19 polymorphism on the eradication rate of Helicobacter pylori infection by 7-day triple therapy with regular proton pump inhibitor dosage. J Gastroenterol Hepatol 23: 1287-1291, 2008. 
70. Perri F, Manes G, Neri M, Vaira D and Nardone G: Helicobacter pylori antigen stool test and ${ }^{13} \mathrm{C}$-Urea breath test in patients after eradication treatments. Am J Gastroenterol 97: 2756-2762, 2002.

71. World Gastroenterology Organisation: World Gastroenterology Organisation Global Guideline: Helicobacter pylori in developing countries. J Clin Gastroenterol 45: 383-388, 2011.

72. Czinn SJ and Blanchard T: Vaccinating against Helicobacter pylori infection. Nat Rev Gastroenterol Hepatol 8: 133-140, 2011.

73. Moss SF, Moise L, Lee DS, et al: HelicoVax: epitope-based therapeutic Helicobacter pylori vaccination in a mouse model. Vaccine 29: 2085-2091, 2011.

74. Othman MO and Wallace MB: Endoscopic mucosal resection (EMR) and endoscopic submucosal dissection (ESD) in 2011 a Western perspective. Clin Res Hepatol Gastroenterol 35: 288-294, 2011.
75. Pandalai PK, Lauwers GY, Chung DC, Patel D and Yoon SS: Prophylactic total gastrectomy for individuals with germline CDH1 mutation. Surgery 149: 347-355, 2011.

76. Wu CY, Wu MS, Kuo KN, Wang CB, Chen YJ and Lin JT: Effective reduction of gastric cancer risk with regular use of nonsteroidal anti-inflammatory drugs in Helicobacter pyloriinfected patients. J Clin Oncol 28: 2952-2957, 2010.

77. Navarro Silvera SA, Mayne ST, Risch HA, et al: Principal component analysis of dietary and lifestyle patterns in relation to risk of subtypes of esophageal and gastric cancer. Ann Epidemiol 21: 543-550, 2011. 